\title{
A Synchronization Scheme for Antenna in Polarization Hopping Transmission System
}

\author{
Zuohong $\mathrm{Xu}{ }^{\text {a }}$, Yanmin Ma ${ }^{\mathrm{b}}$, Qian Cheng ${ }^{\mathrm{c}}$, Jiang Zhu ${ }^{\mathrm{d}}$ and Kai Gao ${ }^{\mathrm{e}}$ \\ University of Defense Technology, Changsha 410000, China. \\ azuohong.xu@outlook.com, bmayanmin16@nudt.edu.cn, cchengqian14a@nudt.edu.cn, \\ djiangzhu@nudt.edu.cn, egaokai000@nudt.edu.cn
}

\begin{abstract}
A polarization hopping wireless communication system is proposed, the matching relationship between polarization hopping sequence and polarization states of antennas is designed, and a synchronization acquisition scheme is put forward for the proposed polarization hopping wireless communication system. Then the detection and false alarm probabilities of the proposed synchronization acquisition scheme are analyzed and simulated under different pseudo-random sequences using MATLAB. The simulated results demonstrate that the proposed system can achieve best synchronization performance with RS sequence, and they also verify the feasibility of the proposed synchronization acquisition scheme.
\end{abstract}

Keywords: Polarization hopping system, matching relationship, synchronization acquisition scheme, detection and false alarm probabilities.

\section{Introduction}

The traditional microwave transmission systems mainly employ the time, frequency or space characteristics of signals to improve transmission efficiency, security or anti-jamming performances. In recent years, it has raised many researchers' concerns to exploit polarization characteristics of signals to further improve the performance of microwave transmission systems. The work in [1-4] presented the multiple polarization transmission, which improved transmission efficiency. [5] studied dual-polarized antennas allowing the transmission/reception from both horizontal and vertical polarized radio signals, thus improving the overall system performance. [6] researched polarization agile antennas and the polarization agile transmission system. Moreover, a simultaneous generation of two microwave signals with a precisely-controlled phase difference using the orthogonal polarization modes in a microwave transmission system was described in [7]. Fast-polarizationhopping transmission diversity was studied to mitigate prolonged deep fades in indoor wireless communications in [8]. Besides, multi-stage quantum secure microwave transmission using polarization hopping was researched by Rifai and et al in [9]. In [10], authors investigated the design and performance on frequency hopping sequence with minimum gap based on RS code.

Overall, there are mainly two ways to utilize polarization characteristics in the present microwave transmission systems: a) using discrete polarization states to carry information bits and improve transmission efficiency; b) applying polarization hopping to improve the security or anti-jamming performance. However, nearly all of present literature only analyzes system's performances theoretically under the assumption that transmitter and receiver antennas can achieve accurate polarization synchronization, thus the polarization synchronization for the polarization hopping system still remains an outstanding problem.

This paper is dedicated to the problem of polarization synchronization for transmitter and receiver antennas based on a polarization hopping microwave transmission system. Firstly, we elaborate a polarization hopping microwave transmission system, where the antennas' polarization states vary with a polarization hopping sequence in a large range rather than being limited to vertically-polarized and horizontally-polarized dipoles. Then, the matching relationship between polarization hopping sequence and polarization states of antennas is designed, and a feasible synchronization acquisition scheme is proposed for the transmitter and receiver antennas. At the end, we analyze and simulate the detection probability and false alarm probability of the proposed acquisition scheme under different pseudo-random sequences. 


\section{System Model}

\subsection{Polarization Hopping System Model.}

The signals transmitted by a polarization-fixed antenna can behave the same polarization characteristics as the antenna. When the polarization state of the receiving antenna is the same as the transmitting antenna, there will be no attenuation in the received signal. On the other hand, when the polarization state between the receiving antenna and the transmitting antenna differs, the mismatch polarization angle will lead to severe attenuation and distortion in the received signal and further exacerbate the demodulating performance. Taking the polarization mismatch principles mentioned above and the idea of frequency hopping in frequency hopping system into consideration, we construct a polarization hopping wireless communication system which is shown in Figure 1.

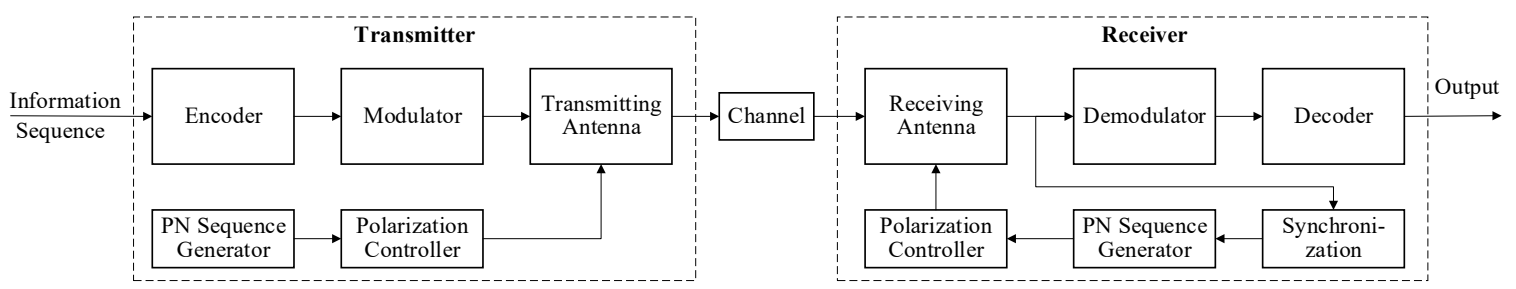

Figure 1. Block Diagram of Polarization Hopping Communication System.

Compared with traditional wireless communication system, the key idea of the proposed polarization hopping communication system is that, the polarization characteristics of transmitting and receiving antennas are controlled by a varying regularity rather than remaining fixed. The varying regularity, which can be a pseudo-random sequence (polarization hopping sequence), is shared between the transmitter and receiver in order to guarantee the synchronization. When the varying regularity of transmitting and receiving antennas synchronizes, the receiver can recover the original modulated signals correctly. However, if the receiver does not know the varying regularity or it knows but not synchronizes with the transmitter, received signals will differ greatly from the original modulated signals.

Evidently, the key problems to realize the polarization hopping communication system lie in three aspects: a) how to choose polarization hopping sequence; b) how to design the matching relationship between polarization hopping sequence and polarization states of antennas; and c) how to structure the polarization synchronization scheme, which will be described as follows.

\subsection{Polarization Synchronization Analysis.}

As mentioned above, polarization synchronization is the key to realize polarization hopping system. Specifically, polarization synchronization contains two kinds of synchronizations: a) the synchronization of polarization hopping sequence and $b$ ) the synchronization of time. This paper mainly focuses on the synchronization of polarization hopping sequence.

Considering the sequential synchronization acquisition method based on energy correlation detection of frequency hopping system, we take the received signal's energy as the acquisition index to construct the synchronization acquisition scheme for the proposed polarization hopping communication system. What's different, there are no detected signals when the frequency hopping sequence doesn't synchronize in the frequency hopping system, while there still exists detected and attenuated signals when the polarization hopping sequence doesn't synchronizes in the polarization hopping system, which poses a more challenging problem to the polarization synchronization in the polarization hopping system. 


\section{Synchronization Acquisition Scheme}

\subsection{Polarization Hopping Sequence.}

Taking into account the particularity of polarization synchronization, polarization hopping sequence should satisfy the following requirements: a) high randomness and linear complexity; b) the gaps between adjacent hops should be as large as possible in order to enhance the difficulty of tracking polarization hopping sequence for the eavesdropper; and c) the hardware circuit that generates the polarization hopping sequence should be as simple as possible.

Once the polarization hopping sequence is determined, we design the matching relationship between polarization hopping sequence and antenna's polarization states expressed in the form of polarization angle in Figure 2, where the quantization bit is assumed to be 8 . In this matching relationship, even hops are distributed in the smaller polarization angle range and odd hops are distributed in the larger polarization angle range. In addition, the larger polarization hop is, the closer to $90^{\circ}$ the corresponding antenna's polarization angle is. Noting the fact that the received signal will attenuate in the largest scale when the mismatch polarization angle between the transmitting and receiving antennas' polarization states equals $90^{\circ}$, thus the advantage of this matching relationship is that the gaps between adjacent hops are large enough that the eavesdropper cannot track the polarization hopping sequence, which can guarantee the eavesdropper's received signal attenuates as large as possible.

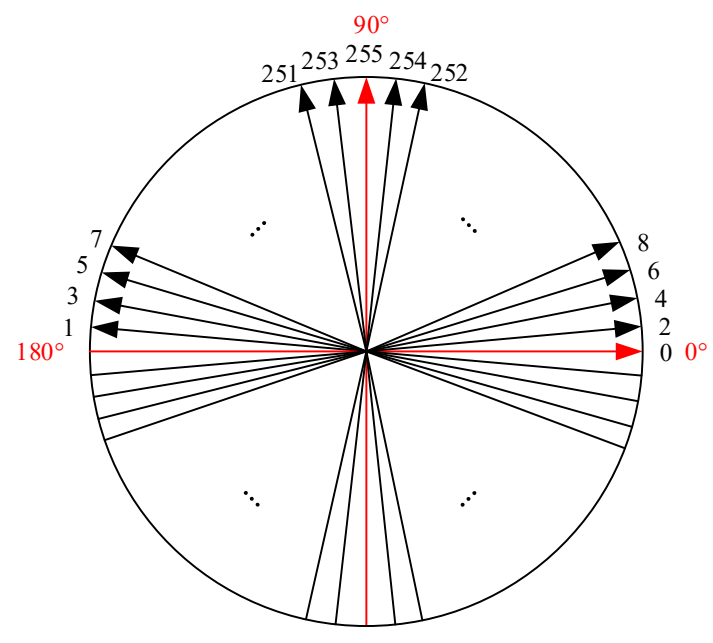

Figure 2. Matching Relationship Between Polarization Hopping Sequence and Antenna'S Polarization States.

\subsection{Synchronization Acquisition Scheme.}

As explained in the last part, when the polarization hopping sequence synchronizes perfectly (that is, the polarization states between the transmitting and receiving antennas are perfectly matched), received signal's energy will reach the peak; and when the polarization hopping sequence doesn't synchronize (that is, the polarization states between the transmitting and receiving antennas are not matched), received signal's energy will attenuate greatly. Thus, we take the received signal's energy as the acquisition index in the proposed synchronization acquisition scheme which is shown in Figure 3. 


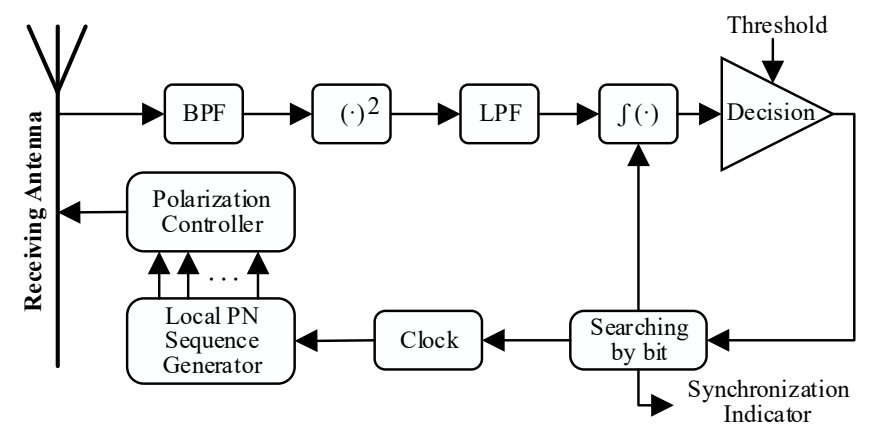

Figure 3. Synchronization Scheme for the Polarization Hopping Sequence.

The local PN sequence generator controls the polarization controller to produce different polarization hopping states of receiving antenna and then the polarization hopping states drive receiving antenna to receive signals. Next, received signals are sent into a band-pass filter (BPF) to filter the out-of-band noise, and the output of BPF is sent into an energy detector. Then, comparing the detected energy with a fixed threshold, if the detected energy is smaller than the threshold, the searching unit will delete a clock pulse when the local PN sequence generator generates the next sequence. The above steps are repeated until the detected energy is larger than the threshold successively for three times, which means the acquisition is achieved. Owing to the undetected errors and false alarm probability, three times of successive decisions are necessarily required.

There are two main differences compared with the sequential searching acquisition method in frequency hopping system: a) what the polarization hopping sequence drives is the polarization controller, rather than the frequency synthesizer in frequency hopping system; b) since the polarization states of receiving antenna are controlled by polarization controller, the receiving antenna needs to be included in the acquisition loop circuit, while it is not required in frequency hopping system.

\subsection{Acquisition Performance Analysis.}

Detection probability and false alarm probability are two main indicators for the acquisition performance. Next, we analyze the detection probability and false alarm probability of the proposed acquisition scheme.

Assume the channel is addictive Gaussian white noise (AWGN) channel. For the receiver, the signal waveform of the i-th polarization hopping interval can be expressed in the narrow-band form as

$$
\mathrm{S}_{\mathrm{R}}(t)=\alpha\left(\Delta \theta_{i}\right) A_{i} \cos \left(\omega_{i} t+\varphi_{i}\right)+n_{i}(t)
$$

where $A_{i}, \omega_{i}$ and $\varphi_{\mathrm{I}}$ are the amplitude, carrier frequency and phase of the i-th signal waveform, respectively; $\Delta \theta_{i}$ is the mismatch polarization angle between transmitting and receiving antennas, and $\alpha\left(\Delta \theta_{\mathrm{i}}\right)$ is the corresponding normalized amplitude attenuation.

Furthermore, the polarization hopping interval can be transformed into the amplitude-angle form as

Where

$$
S_{R}(\mathrm{t})=R_{i}(t) \cos \left[\omega_{i} \mathrm{t}+\varphi_{\mathrm{i}}+\vartheta_{i}(t)\right.
$$

$$
\begin{gathered}
R_{i}(t)=\sqrt{\left[\alpha\left(\Delta \theta_{i}\right) A_{i}+n_{c}(t)\right]^{2}+n_{s}^{2}(t)}, \\
\vartheta_{i}(t)=\arctan \frac{n_{s}(t)}{\alpha\left(\Delta \theta_{i}\right) A_{i}+n_{c}(t)}
\end{gathered}
$$


and $R_{i}(t)$ is a random variable with Rician distribution; $n_{i}(t), n_{c}(t)$ and $n_{s}(t)$ are narrowband Gaussian noises, whose variances are $\sigma^{2}=N_{0} B / 2$ where $N_{0}$ is single-side power spectral density and $\mathrm{B}$ is the bandwidth of BPF.

Assume the length of integral intervals is $\mathrm{M}$, and the output of energy detector is $\mathrm{Z}$, that is, $\mathrm{Z}=$ $\sum_{i=1}^{M} R_{i}^{2}(t)$, which is the quadratic sum of $\mathrm{M}$ random variables with Rician distribution, and it follows the $2 \mathrm{M}$-degree-of-freedom noncentral chi square distribution whose probability density function is

$$
P_{Z}(\varepsilon)=\left\{\begin{array}{cc}
\frac{1}{2}\left(\frac{\varepsilon}{s}\right)^{\frac{M-1}{2}} \exp \left(-\frac{s+\varepsilon}{2}\right) I_{M-1}(\sqrt{s \varepsilon}), \varepsilon>0 \\
0, \quad \text { o.w. }
\end{array}\right.
$$

Where $\varepsilon=\mathrm{z} / \sigma^{2}, I_{M-1}(x)$ is the $(\mathrm{M}-1)$-th order modified Bessel function, and $\mathrm{s}$ is the normalized noncentral parameter of the noncentral chi square distribution whose expression is

$$
\mathrm{s}=\sum_{i=1}^{M}\left[\alpha\left(\Delta \theta_{i}\right) A_{i}\right]^{2} / \sigma^{2}
$$

According to the equation (5), we can obtain the detection probability (the probability decided as synchronization when the polarization hopping sequence matches) as

$$
P_{d}=P\left(z>\varepsilon_{T} \mid \Delta \theta_{i}=0, i=1,2 \ldots, M\right)=\int_{\varepsilon_{0}}^{\infty} p_{z}\left(\varepsilon \mid \Delta \theta_{i}=0, i=1,2, \ldots, M\right) d \varepsilon
$$

Where $\varepsilon_{T}$ is the threshold and $\varepsilon_{0}=\varepsilon_{T} / \sigma^{2}$ is the normalized threshold. Taking equations (5) and (6) into (7), the detection probability can be simplified as

Where

$$
P_{d}=Q_{M}\left(\sqrt{s_{d}}, \sqrt{\varepsilon_{0}}\right)
$$

$$
s_{d}=\left.s\right|_{\Delta \theta_{i}=0, i=1,2, \ldots, M}=\sum_{i=1}^{M} A_{i}^{2} / \sigma^{2},
$$

And $Q_{M}(a, b)$ is the Marcum Q-function, that is,

$$
Q_{M}(a, b)=\int_{a}^{\infty} x\left(\frac{x}{a}\right)^{M-1} \exp \left(-\frac{x^{2}+a^{2}}{2}\right) I_{M-1}(a x) d x
$$

The fact that the received signal's normalized amplitude is 1 when the polarization hopping sequence synchronizes perfectly, that is, $\left.\alpha\left(\Delta \theta_{i}\right)\right|_{\Delta \theta_{i}=0}=1$, has been applied in the equation (9).

Similarly, the false alarm probability (the probability decided as synchronization when the polarization hopping sequence mismatches) can be expressed as

$$
P_{f a}=P\left(z>\varepsilon_{T} \mid \Delta \theta_{i} \neq 0, i=1,2, \ldots M\right)=\int_{\varepsilon_{0}}^{\infty} p_{z}\left(\varepsilon \mid \Delta \theta_{i} \neq 0, i=1,2, \ldots, M\right) d \varepsilon
$$

Taking equations (5) and (6) into (11), we can acquire the simplified false alarm probability as

Where

$$
P_{f a}=Q_{M}\left(\sqrt{s_{f a}}, \sqrt{\varepsilon_{0}}\right)
$$

$$
s_{f a}=\left.s\right|_{\Delta \theta_{i} \neq 0, i=1,2, \ldots, M}=\sum_{i=1}^{M}\left[\alpha\left(\Delta \theta_{i}\right) A_{i}\right]^{2} / \sigma^{2} .
$$

\section{Simulation Results}

Firstly, taking microstrip antenna model into consideration, we simulate the mapping relationship between the power loss of received signal and polarization mismatch angle which is shown in Table 
1 , where the angle interval is $5^{\circ}$, and the received signal's power is normalized to be $1(0 \mathrm{~dB})$ when the mismatch angle is zero.

Table 1. Simulated Power Loss of Received Signal Versus Mismatch Polarization Angle.

\begin{tabular}{|c|c|c|c|c|c|}
\hline $\begin{array}{c}\text { Mismatch Angle } \\
\left({ }^{\circ}\right)\end{array}$ & $\begin{array}{c}\text { Power Loss } \\
(\mathrm{dB})\end{array}$ & $\begin{array}{c}\text { Mismatch } \\
\text { Angle }\end{array}$ & $\begin{array}{c}\text { Power Loss } \\
(\mathrm{dB})\end{array}$ & $\begin{array}{c}\text { Mismatch } \\
\text { Angle }\end{array}$ & $\begin{array}{c}\text { Power Loss } \\
(\mathrm{dB})\end{array}$ \\
\hline 0 & 0 & 35 & 1.73 & 65 & 7.48 \\
\hline 5 & 0.03 & 40 & 2.31 & 70 & 9.32 \\
\hline 10 & 0.13 & 45 & 3.01 & 75 & 11.74 \\
\hline 15 & 0.30 & 50 & 3.84 & 80 & 15.21 \\
\hline 20 & 0.54 & 55 & 4.83 & 85 & 21.19 \\
\hline 25 & 0.85 & 60 & 6.02 & 90 & 30.00 \\
\hline 30 & 1.25 & & & & \\
\hline
\end{tabular}

According to our proposed acquisition scheme, we simulate the acquisition performances when the system adopts $m$ sequence, Gold sequence and RS sequence as the polarization hopping sequence respectively. The length of above three pseudo-random sequences is $2^{8}-1=255$. Besides, both of the primitive polynomials of $m$ sequence and RS sequence are $f_{1}(x)=1+x^{3}+x^{5}+x^{6}+x^{8}$, and the two primitive polynomials of Gold sequence are $f_{1}(x)$ and $f_{2}(x)=1+x^{1}+x^{3}+x^{5}+x^{8}$. The original values of shift registers are set as 00000001, and QPSK modulation is adopted, which means the amplitude in (1) is a constant, that is, $A_{i}=A(i=1,2, \ldots, M)$.

The simulated result shows that the received signal's normalized energy reaches the peak 1 when the polarization hopping sequence synchronizes perfectly; and the received signal's normalized energy is around 0.5 when the polarization hopping sequence doesn't synchronize. Therefore, as long as the threshold is chosen properly, the proposed acquisition scheme will be achieved feasibly and practically.

Table 2. Variance of Received Signal's Normalized Energy with Different Sequences $\left(\times 10^{-3}\right)$.

\begin{tabular}{|c|c|c|c|}
\hline & $\mathrm{M}=20$ & $\mathrm{M}=40$ & $\mathrm{M}=60$ \\
\hline m sequence & 12.075 & 6.812 & 4.915 \\
\hline Gold sequence & 11.389 & 7.306 & 6.052 \\
\hline RS sequence & 9.651 & 5.134 & 3.844 \\
\hline
\end{tabular}

Besides, Table 2 shows the variances of received signal's normalized energy with different sequences the received signal's normalized energy in the non-synchronization case, where the number of integral intervals is set as $M=20,40$ and 60 . We can see that the variance of received signal's normalized energy with RS sequence adopted is always the smallest when the number of integral intervals is set as the same value, which means RS sequence is better than m sequence and Gold sequence for the proposed acquisition scheme, and RS sequence can better satisfy the requirements of synchronization sequence.

Figure 4 and Figure 5 depict the detection probability versus signal-to-noise power ratio (SNR) with different number of integral intervals and different false alarm probability. Apparently, the detection probability increases as the number of integral intervals increases when the false alarm probability is fixed, and it also increases as the false alarm probability increases when the number of integral intervals is fixed. In fact, the complexity of practical hardware circuit is directly proportional to the number of integral intervals so that the setting of integral intervals and threshold should compromise between high detection probability and low hardware complexity. 


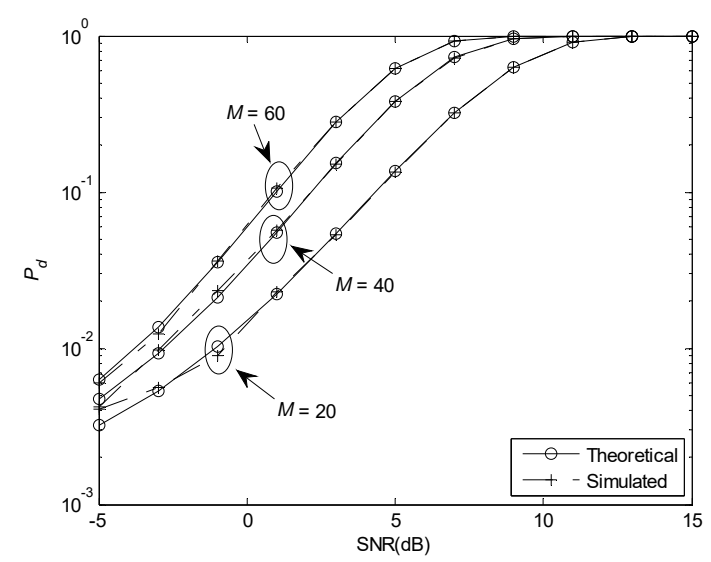

Figure 4. Detection Probability Versus SNR

$$
\left(P_{f a}=10^{-3}\right) \text {. }
$$

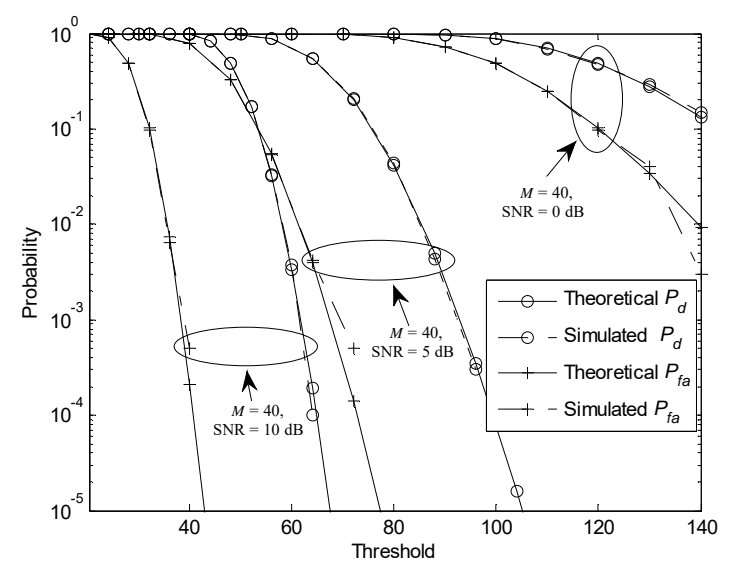

Figure 6. Detection Probability Versus SNR $\left(P_{f a}=10^{-3}\right)$

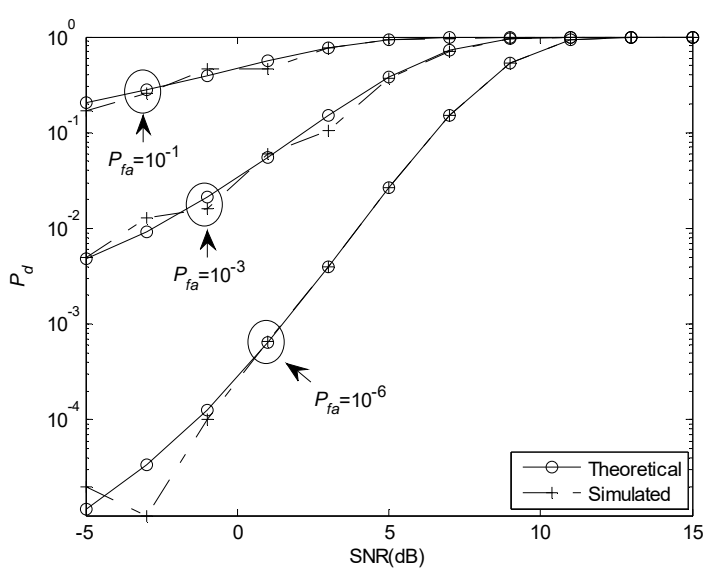

Figure 5. Detection Probability Versus SNR $(\mathrm{M}=40)$.

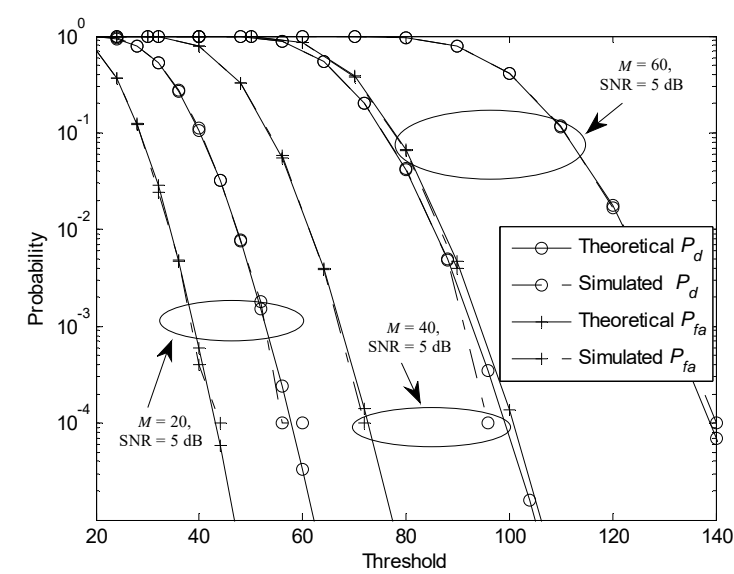

Figure 7. Detection Probability Versus SNR

$$
\left(P_{f a}=10^{-3}\right)
$$

Figure 6 and Figure 7 show the detection and alarm probabilities versus the threshold and different SNR. Now that the higher $P_{d}$ is and the lower $P_{f a}$ is, the better acquisition performance will be achieved. From Figure 6, we can set the threshold properly with a given $P_{d}$ and $P_{f a}$. For example, when $\mathrm{M}=40$ and $\mathrm{SNR}=10 \mathrm{~dB}$, in order to achieve the performance of $P_{d} \rightarrow 1$ and $P_{f a} \approx 10^{-4}$, the threshold should be set as $\varepsilon_{T}=40$, which can provide useful advice in the practical appliance of the proposed acquisition.

\section{Conclusion}

In this paper, we construct a polarization hopping microwave transmission system, design the matching relationship between polarization hopping sequence and polarization characteristics of antennas, and propose a synchronization acquisition scheme for the transmitter and receiver antennas. Besides, the detection and false alarm probabilities of proposed acquisition scheme are analyzed and simulated. The simulated results demonstrate that the proposed system can achieve best synchronization performance with RS sequence adopted, and they also verify the feasibility of the proposed synchronization acquisition scheme. 


\section{References}

[1]. R. B. Dybdal, S. J. Curry, F. Lorenzelli, et al. Multiple Polarization Communications. Proc. IEEE International Symposium on Antennas and Propagation, Chicago, IL, USA, July, 2012, p. $1-2$.

[2]. K. R. Mahmoud, A. M. Montaser. Performance of tri-band multi-polarized array antenna for 5G mobile base station adopting polarization and directivity control. IEEE Access. Vol.6 (2018), p. 2169-3536.

[3]. C. Meng, J. Shi, J. X. Chen. Flat-gain dual-patch antenna with multi-radiation nulls and low cross-polarization. Electronic Letters. Vol.54 (2018), No. 3, p. 114-116.

[4]. D. Gooshchin, A. Gooshchin. Multi-polarization antenna system with a spherical radiation pattern. IEEE International Conference on Microwaves, Antennas, Communications and Electronic Systems. Tel-Aviv, Israel, 2017. p. 1-4.

[5]. F. Robol, G. Gottardi, M. Salucci, et al. Design of dual-polarization antennas for 5G base stations. IEEE International Symposium on Antennas and Propagation \& USNC/URSI National Radio Science Meeting. San Diego, CA, USA, 2017, p. 2625-2626.

[6]. M.D. Lockard. Analysis of a polarization agile communication system. IEEE Aerospace Conference. Big Sky, MT, USA, June 2015, p. 1-7.

[7]. K. T. Wong, S. L. Chan, R. P. Torres. Fast-Polarization-Hopping Transmission Diversity to Mitigate Prolonged Deep Fades in Indoor Wireless Communications. IEEE Antennas and Propagation Magazine. Vol.48 (2006), No.3, p. 20-27.

[8]. Claude Oestges. Indoor Wireless Communications with Multiple Antennas and Polarizations: From Channel Characterization to Performance Simulation. IEEE International Symposium on Personal, Indoor \& Mobile Radio Communications, Cannes, France, Sep. 2008, p. 1-6.

[9]. M.E. Rifai, K. Chan, P. K. Verma. Multi-stage quantum secure communication using polarization hopping. Security Comm. Networks. Vol. 8 (2016), No. 18, p. 4333-4342.

[10]. G. $\mathrm{Wu}, \mathrm{D}$. Wang. Design and performance investigation on frequency hopping sequence with minimum gap based on RS code. International Conference on Electrical \& Control Engineering. Wuhan, China, June 2010, p. 5120-5123. 